PlastOx 2007 (2009) 131-145

(C) EDP Sciences, 2009

DOI: $10.1051 /$ ptox/2009010

\title{
L’hydrogène dans les matériaux métalliques en relation avec les interactions plasticité-environnement
}

\author{
J. Chêne
}

CEA/CNRS, UMR 8587, Lab. d'Étude de la Corrosion Aqueuse, Bât. 458, CEA Saclay, 91191 Gif-sur-Yvette, France

e-mail : jacques.chene@cea.fr

\begin{abstract}
Résumé. L'objectif de cet article est de faire une revue synthétique des données de la littérature qui permettent de caractériser le comportement de l'hydrogène dans les matériaux métalliques et plus particulièrement les effets d'endommagement associés à l'hydrogène en relation avec les interactions plasticité-environnement. Dans cette présentation générale nous nous sommes limités au comportement de l'hydrogène dans les matériaux de structure tels que les alliages base fer et base nickel.

Les principaux points abordés dans cette présentation sont :

- des généralités sur l'absorption de l'hydrogène dans ces alliages : diffusion, perméation, piégeage,

- une courte revue des principaux effets d'endommagement associés à l'absorption d'hydrogène,

- quelques remarques spécifiques aux interactions hydrogène-plasticité.
\end{abstract}

\section{INTRODUCTION}

De nombreux paramètres d'ordre mécanique, métallurgique, physico-chimique, électrochimique,..., sont à prendre en compte dans la dégradation fréquente des propriétés mécaniques des matériaux exposés à un environnement donné. Une des principales difficultés associées à l'identification des mécanismes et à la prédiction et la prévention de l'endommagement, réside dans le caractère local des phénomènes mis en jeu qui rend souvent très difficile l'identification des paramètres pertinents et la mesure des grandeurs critiques. Il est cependant bien admis maintenant que la connaissance des interactions plasticité-environnement est déterminante dans la compréhension des mécanismes d'endommagement. Cela impose donc une connaissance précise, au niveau local, de la plasticité du matériau, de la nature et l'agressivité de l'environnement et des espèces mises en jeu, et surtout d'une possible synergie des interactions plasticité-environnement.

Il est bien connu que l'hydrogène occupe une place particulière dans les phénomènes d'endommagement des matériaux soumis à des sollicitations mécaniques en présence d'un environnement. En effet l'hydrogène est, de façon quasi inévitable, soit présent à l'état gazeux à l'interface matériau/environnement, soit généré par des réactions physico-chimiques souvent associées à la décomposition de l'eau (dissociation de la vapeur d'eau, décharge cathodique associée à un processus de corrosion humide ou de dépassivation/repassivation, ....). L'hydrogène est par ailleurs le seul élément susceptible, à température modérée, de pénétrer et diffuser sur de longues distances dans les matériaux métalliques. Il peut ainsi, dès la température ambiante, affecter en volume les propriétés mécaniques d'un matériau. Ces différentes caractéristiques expliquent par exemple l'effet néfaste important de la vapeur d'eau présente dans l'atmosphère, sur les propriétés mécaniques de matériaux métalliques soumis à des sollicitations cycliques, ou encore l'observation de phénomènes extrêmement rapides de rupture différée de matériaux exposés à des environnements agressifs.

Le terme de fragilisation par l'hydrogène est souvent utilisé pour décrire certaines formes d'endommagement dans lesquels le rôle de l'hydrogène est clairement identifié même si les mécanismes mis en jeu ne sont pas encore toujours bien compris. Il existe d'autres formes d'endommagement importantes, telles que la corrosion sous contrainte (CSC) qui associe plusieurs phénomènes (oxydation, corrosion, absorption d'hydrogène). Dans certains phénomènes de CSC le rôle de l'hydrogène est 
prédominant, dans d'autres, sans jouer un rôle direct, il peut contribuer au processus d'endommagement. Par exemple, lors de la fissuration intergranulaire par CSC à haute température des alliages base nickel, si l'existence d'un effet fragilisant de l'hydrogène reste à démontrer il pourrait cependant contribuer à l'amorçage/propagation d'une fissure en affectant différentes étapes possibles du processus (plasticité locale, glissement intergranulaire, diffusion de l'oxygène, ....).

Une analyse prédictive fiable du comportement d'un matériau dans son environnement impose donc de bien comprendre le rôle joué par chacun des phénomènes ou espèces impliqués. En ce qui concerne l'hydrogène, il est important de disposer de données précises d'ordre thermodynamique et cinétique sur son absorption, sa diffusion, sa localisation et son interaction avec les défauts microstructuraux du matériaux. De nombreux travaux récents ont montré l'importance des interactions hydrogène-plasticité et notamment le rôle de l'hydrogène sur le mouvement des dislocations auquel peut être couplé un transport d'hydrogène associé aux dislocations mobiles.

Cependant de nombreuses questions restent posées et l'acquisition de données expérimentales précises couplée à des travaux de modélisation est indispensable. Cela concerne aussi bien le comportement de l'hydrogène en proche surface, en relation avec l'état local de contrainte/déformation, que les interactions hydrogène-plasticité en volume (comportement collectif $\mathrm{H}$-dislocations, piégeage dynamique, ...).

Après une présentation générale du comportement de l'hydrogène dans des matériaux de structure tels que les aciers et les alliages base Ni nous aborderons brièvement quelques aspects des interactions H-plasticité en soulignant les points qui mériteraient d'être approfondis.

\section{GÉNÉRALITÉS SUR L'ABSORPTION DE L'HYDROGÈNE DANS LES ACIERS ET LES ALLIAGES BASE NICKEL}

\subsection{Principales caractéristiques de l'hydrogène}

Les grandeurs rassemblées dans le tableau 1 [1] permettent quelques commentaires généraux sur le comportement de l'hydrogène dans les aciers.

L'hydrogène est le plus petit des atomes ; son rayon atomique, du même ordre de grandeur que la taille des sites interstitiels du réseau métallique, lui permet d'entrer facilement en solution d'insertion dans le fer et les aciers. Les sites interstitiels privilégiés sont les sites tétraédriques pour la structure cubique centrée et octaédriques pour la structure cubique à faces centrées.

Cependant le volume molaire partiel relativement élevé de l'hydrogène indique que son introduction dans le fer induit une distorsion notable du réseau qui pourra conduire à la formation de défauts dans le

Tableau 1. Quelques grandeurs caractéristiques relatives à l'hydrogène.

Données physiques

\begin{tabular}{|l|}
\hline Trois isotopes $:{ }_{1}^{1} \mathrm{H},{ }_{1}^{2} \mathrm{H},{ }_{1}^{3} \mathrm{H}$ \\
\hline Masse molaire $\mathrm{H}_{2}: 2,016 \mathrm{~g}$ \\
\hline $1 \mathrm{~cm}^{3} \mathrm{H}_{2}=5,38 \times 10^{19}$ atomes \\
\hline$d_{\mathrm{M}-\mathrm{H}} \approx 1,5 \times 10^{-1} \mathrm{~nm}: d_{\mathrm{H}-\mathrm{H}} \approx 0,74 \times 10^{-1} \mathrm{~nm} ; r_{\mathrm{H}} \approx 0,53 \times 10^{-1} \mathrm{~nm}$ \\
\hline Volume molaire partiel $: \overline{\mathrm{V}}_{\mathrm{H}}=2 \mathrm{~cm}^{3} / \mathrm{mol}$ \\
\hline Mobilité $: D_{\mathrm{HF}} \alpha \approx 9 \times 10^{-5} \mathrm{~cm}^{2} \cdot \mathrm{s}^{-1} \dot{\mathrm{a}} 20^{\circ} \mathrm{C}$ \\
\hline Taille des sites interstitiels \\
\hline $\mathrm{Fe} \alpha\langle\mathrm{cc}\rangle \quad$ Sites tétraédriques $: r_{\mathrm{i}} \approx 3,7 \times 10^{-2} \mathrm{~nm}$ \\
\hline Sites octaédriques: $r_{\mathrm{i}} \approx 1,9 \times 10^{-2} \mathrm{~nm}$ \\
\hline $\mathrm{Fe} \gamma\langle\mathrm{cc}\rangle \quad$ Sites tétraédriques $: r_{\mathrm{i}} \approx 2,9 \times 10^{-2} \mathrm{~nm}$ \\
\hline \multicolumn{2}{c|}{ Sites octaédriques: $r_{\mathrm{i}} \approx 5,2 \times 10^{-2} \mathrm{~nm}$} \\
\hline$r_{\mathrm{H}}:$ rayon atomique de l'hydrogène \\
\hline$r_{\mathrm{i}}:$ rayon des sites interstitiels \\
\hline$d:$ distance interatomique \\
\hline
\end{tabular}


matériau, d'autant plus facilement que la répartition de l'hydrogène sera hétérogène. Parallèlement, la présence de champs de déformation imposera, dans le matériau, une distribution hétérogène de l'hydrogène qui se localisera préférentiellement dans les zones soumises à des contraintes hydrostatiques positives (dilatation du réseau).

De par sa taille (volume d'activation négligeable), sa masse (fréquence de saut élevée) et l'existence d'une forte densité de sites interstitiels disponibles, la mobilité de l'atome d'hydrogène est très grande dans les aciers. Bien que fortement dépendante de la structure cristalline elle est, à la température ambiante, supérieure d'environ 10 ordres de grandeur à celle des autres interstitiels [2]. L'hydrogène est en fait le seul élément pour lequel les vitesses de diffusion sont directement mesurables à température ambiante. La valeur de son coefficient de diffusion correspond à un parcours quadratique moyen de l'ordre de $1 \mathrm{~mm}$ en 1 minute à $20^{\circ} \mathrm{C}$ dans le fer $\alpha$ et explique sa très grande aptitude à pénétrer dans les aciers ferritiques.

L'aptitude de l'hydrogène interstitiel à transférer tout ou partie de sa charge électronique aux atomes proches voisins est à l'origine de son rôle particulier sur la cohésion des réseaux métalliques et notamment de son effet fragilisant. L'électron 1 s associé au proton interagit avec les bandes s et d du métal modifiant ainsi la densité d'état au niveau de Fermi et favorisant un déplacement des niveaux d'énergie [3]. Cette modification de la structure électronique locale des atomes de métal, combinée à une augmentation de la distance interatomique, affecte directement les forces de cohésion du réseau. Dans le cas des métaux de transition tels que le fer, cet effet se traduit par une diminution des forces de cohésion [4].

La solubilité de l'hydrogène dans un métal est étroitement liée à son interaction électronique avec les atomes du réseau et donc à la structure électronique du métal considéré. Elle est généralement faible pour les métaux de transition mais elle dépend également de la structure cristalline du matériau (cf. $\S 2.3$ ). Cette faible solubilité favorise la migration de l'hydrogène interstitiel vers des sites microstructuraux (défauts, interfaces, impuretés, ... ) énergétiquement plus favorables, communément appelés "pièges".

Ces quelques caractéristiques :

- faible solubilité, donc tendance à la ségrégation,

- forte mobilité, donc aptitude à la ségrégation,

- grand volume partiel molaire, donc sensibilité à la présence de champs de contrainte,

- forte interaction électronique, donc aptitude à une modification de l'environnement électronique des atomes du métal,

sont à l'origine des phénomènes de piégeage et constituent la base des propriétés fragilisantes de l'hydrogène présent à température modérée dans les alliages base fer et nickel.

\subsection{Sources d'hydrogène associées aux interactions plasticité-environnement}

D’une façon générale l'entrée de l'hydrogène dans un métal résulte de sa mise en contact avec différentes sources possibles d'hydrogène : adsorption de molécules $\mathrm{H}_{2}$, décharge de protons ou d'espèces chargées issues d'un électrolyte ou d'un plasma, décomposition d'espèces hydrogénées à la surface du métal, ... La déformation plastique d'un matériau peut favoriser l'absorption d'hydrogène de différentes façons : augmentation de la réactivité de surface par formation de surfaces métalliques fraîches, rupture de film d'oxyde barrière, modification de l'énergie de surface et création de défauts superficiels ou volumiques favorisant l'absorption de $\mathrm{H}$. De l'hydrogène interne peut préexister dans le matériau suite à diverses opérations (élaboration du métal, soudage,...), il peut également être formé in situ sous l'effet de réactions nucléaires.

Les différents états possibles de l'hydrogène dans les aciers dépendent de son activité, de la température, et de la microstructure du matériau. A l'équilibre sous une pression modérée l'hydrogène est en solution solide à l'état atomique ; sa concentration suit la loi de Sieverts (cf.§ 2.3). Le modèle du 


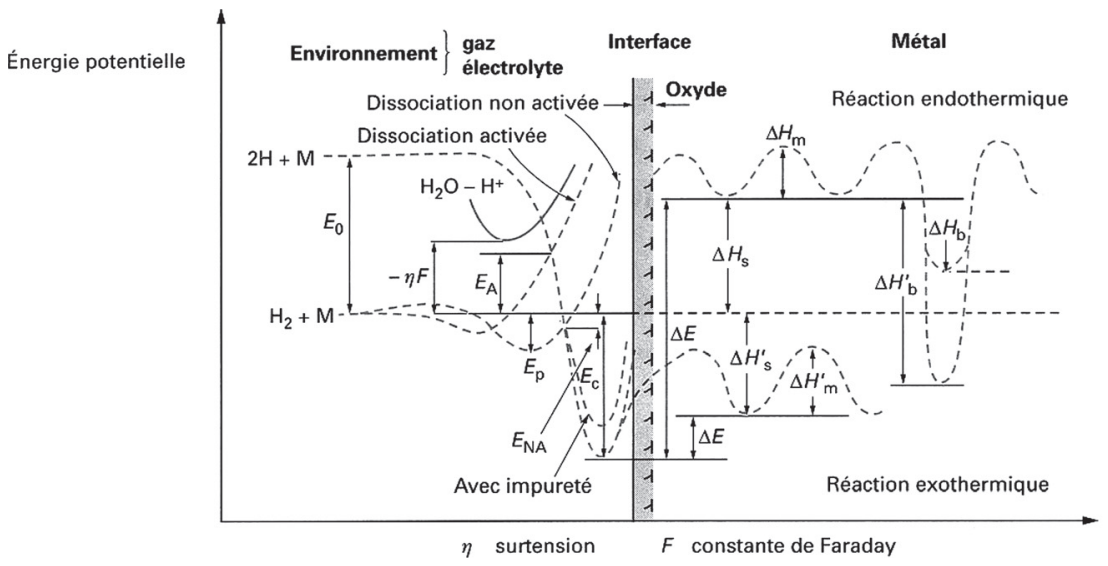

Figure 1. Représentation schématique des différentes étapes et niveaux d'énergie associés à l'entrée de l'hydrogène dans un métal.

proton écranté par les électrons de conduction du métal permet alors de décrire l'état de $\mathrm{H}$ interstitiel dans les aciers [5].

Le piégeage de l'hydrogène résulte de son interaction attractive avec des sites énergétiquement plus favorables que les sites interstitiels ; il s'accompagne d'une diminution de sa mobilité qui est fonction de l'énergie de piégeage. Il correspond à différents états possibles :

- piégeage d'atomes sur les défauts microstructuraux (interaction élastique ou électronique),

- adsorption d'atomes ou de molécules sur les surfaces ou interfaces,

- recombinaison moléculaire sur des défauts (fissures, cavités,...) lorsque l'activité de l'hydrogène dans le réseau est élevée,

- état combiné avec des impuretés (formation de $\mathrm{CH}_{4}, \mathrm{NH}_{3}, \ldots$ ) à pression et température élevées.

\subsection{Absorption et solubilité de l'hydrogène}

Les interactions de l'hydrogène avec la surface métallique seront présentées en détail dans le cadre de cette école [6]. Nous nous limiterons ici à une présentation générale des étapes successives d'adsorption sur la surface, de dissociation et de diffusion en volume [7] qui sont représentées schématiquement sur la figure 1. Le transfert à l'interface est un mécanisme complexe qui fait intervenir des sites présentant différents niveaux d'énergie (surface, subsurface, volume) fonction de la structure et des propriétés physico-chimiques de la surface. Il peut cependant, de façon schématique, être associé au transfert de l'atome d'hydrogène d'un site d'adsorption chimique à un site interstitiel et correspond à une augmentation d'énergie potentielle $(\Delta \mathrm{E})$ plus ou moins grande selon la nature du métal. La valeur et le signe de l'enthalpie de dissolution $\left(\Delta \mathrm{H}_{s}\right)$ caractérise la nature exothermique ou endothermique de la réaction de dissolution; cette dernière est endothermique dans le cas des alliages base fer et nickel [5]. L'hydrogène peut alors diffuser par saut entre sites interstitiels proches voisins selon un processus thermiquement activée $\left(\Delta \mathrm{H}_{\mathrm{m}}\right)$ dans le domaine usuel de température $\left(\mathrm{T}>-50^{\circ} \mathrm{C}\right)$.

\subsubsection{Pénétration à partir d'un environnement gazeux}

L'adsorption dissociative constitue la première étape de l'entrée de l'hydrogène gaz. La cinétique de dissociation est généralement rapide à la surface des métaux de transition tels que Fe et Ni. En revanche une forte énergie d'activation est nécessaire lorsque le métal est recouvert d'une couche d'oxyde ; la pénétration d'hydrogène est dans ce cas contrôlée par la cinétique très lente de dissociation de la 
molécule [8, 9]. Cet effet "barrière" associé à la présence de couche d'oxyde ou d'espèces physiou chimisorbées est un paramètre important à prendre en considération dans les études de diffusion d'hydrogène gazeux ; il est particulièrement sensible aux interactions plasticité-environnement. Il peut être amplifié par une diffusion ralentie au travers de la couche d'oxyde [10].

L'interaction métal-hydrogène gaz peut être schématisée par la réaction :

$$
2 \mathrm{M}+\mathrm{H}_{2} \rightarrow 2 \mathrm{MH}_{\mathrm{abs}}+\mathrm{Q}
$$

La quantité $\mathrm{Q}$ caractérise, selon son signe, le caractère exothermique ou endothermique de la réaction. Dans l'hypothèse d'une solution diluée et d'un taux de recouvrement faible, la solubilité de l'hydrogène dissous s'exprime dans un domaine donné de température et de pression d'hydrogène par une loi de Sieverts :

$$
\mathrm{S}=\mathrm{S}_{\mathrm{o}} \mathrm{p}^{1 / 2} \exp \left(\frac{-\Delta \mathrm{H}_{\mathrm{s}}}{\mathrm{kT}}\right)
$$

enthalpie de dissolution, $\mathrm{p}$ : pression, $\mathrm{T}$ : température.

A noter que l'adsorption d'impuretés sur la surface peut jouer un rôle déterminant sur la pénétration effective d'hydrogène dans le métal [11-13].

\subsubsection{Pénétration à partir d'un milieu liquide}

Tout mécanisme de corrosion électrochimique implique l'existence à l'interface électrolyte-électrode de réactions anodique et cathodique couplées [14]. Dans un environnement source de protons la réaction cathodique a une très forte probabilité de conduire à une décharge de $\mathrm{H}$ sans étape de dissociation selon les réactions du type :

$$
\begin{gathered}
\mathrm{H}^{+}+\mathrm{e}^{-}+\mathrm{M} \rightarrow \mathrm{MH}_{\text {ads }} \text { (milieu acide) } \\
\mathrm{H}_{2} \mathrm{O}+\mathrm{e}^{-}+\mathrm{M} \rightarrow \mathrm{MH}_{\mathrm{ads}}+\mathrm{OH}^{-} \text {(milieu basique) }
\end{gathered}
$$

L'oxyde superficiel peut cependant, en fonction de sa stabilité et de ses propriétés électroniques, jouer un rôle sur le transfert électronique au travers de la double couche et par conséquent sur l'absorption d'hydrogène [15]. La vitesse de réaction est fonction de la surtension hydrogène caractéristique des conditions d'essais et du métal [16].

\subsubsection{Mécanismes de décharge cathodique}

La décharge cathodique de $\mathrm{H}$ et son ad/absorption ont fait l'objet de nombreuses études [17-19]. Elle dépend de différents paramètres : nature de l'électrolyte [20], nature et état structural du métal [21, 22], conditions de polarisation,... Elle fait intervenir trois étapes principales :

- le transfert électronique au travers de la double couche et l'adsorption chimique :

$$
\mathrm{H}^{+}+\mathrm{e}^{-} \rightarrow \mathrm{H}_{\mathrm{ads}}
$$

- la recombinaison des atomes adsorbés qui conduit au dégagement d' $\mathrm{H}_{2}$ :

$$
\mathrm{H}_{\mathrm{ads}}+\mathrm{H}_{\mathrm{ads}} \rightarrow \mathrm{H}_{2}
$$

- la pénétration dans le métal :

$$
\mathrm{M}+\mathrm{H}_{\mathrm{ads}} \rightarrow \mathrm{MH}_{\mathrm{abs}}
$$

Les vitesses relatives de chacune de ces étapes conditionnent l'absorption d'hydrogène et dépendent, à température donnée, de trois paramètres principaux : le taux de recouvrement en hydrogène adsorbé, 
l'énergie d'adsorption et la surtension hydrogène [16]. Comme dans le cas d'un environnement gazeux, la présence d'impuretés sur la surface peut affecter la cinétique des réactions de décharge et recombinaison et contrôler la pénétration d'hydrogène dans le métal [23, 24].

\subsubsection{Pression équivalente associée à l'hydrogénation cathodique}

Pour un mécanismes de décharge donné, la concentration en hydrogène absorbé en surface sera fonction de la fugacité associée aux conditions d'hydrogénation qui, dans l'hypothèse d'un équilibre entre l'hydrogène adsorbé et l'hydrogène dissous s'exprime sous la forme [16] :

$$
\mathrm{f}_{\mathrm{H} 2}=k \exp \left(\frac{\alpha \eta F}{R T}\right)
$$

$\mathrm{k}$ et $\alpha$ : constantes fonction des cinétiques de décharge et recombinaison, $\eta$ : surtension, $\mathrm{F}:$ constante de Faraday.

Dans certaines conditions (exemple du fer en milieu acide à forte surtension cathodique), des valeurs de fugacité très élevées (plusieurs milliers d'atmosphère) peuvent être calculées à température ambiante et convertie en valeur de pression équivalente [25]. De telles valeurs peuvent être mesurées directement à l'aide de la technique de perméation électrochimique [15] si la valeur du coefficient de diffusion de l'hydrogène dans le matériau est connue. Il suffit pour cela de comparer les valeurs de flux stationnaire obtenues à l'aide d'essais de perméation électrochimique à ceux obtenus en phase gazeuse.

A noter que selon les conditions électrochimiques ( $\mathrm{pH}$, mécanisme de décharge, ....) et la chimie locale, l'exposition d'un matériau au potentiel libre peut être associée à des pressions équivalentes élevées qui conduisent à l'absorption de quantités importantes d'hydrogène.

\subsubsection{Données expérimentales sur la solubilité de l'hydrogène dans les aciers et les alliages base Ni}

Le tableau 2 rassemble quelques lois de variation, en fonction de la pression et de la température, de la solubilité de l'hydrogène dans le fer $\alpha$, les aciers inoxydables austénitiques, le nickel et un alliage base Ni. Les valeurs calculées à température ambiante montrent une plus faible solubilité dans le fer $\alpha$ que dans la structure cfc des aciers austénitiques [27]. Cependant les mesures expérimentales conduisent à des valeurs de concentration souvent beaucoup plus élevées en raison de phénomènes de piégeage [28].

\subsubsection{Effet d'un champ de contrainte}

La valeur élevée du volume molaire partiel $\bar{V}_{\mathrm{H}}$ de l'hydrogène dans le fer (tableau 1) conduit à une dépendance de la solubilité de l'hydrogène vis à vis d'un champ de contraintes élastiques qui s'exprime par la relation :

$$
\frac{\mathrm{S}_{\sigma \mathrm{H}}}{\mathrm{S}_{\mathrm{o}}}=\exp \left(\frac{\sigma_{H} \bar{V}_{H}}{R T}\right)
$$

$\sigma_{\mathrm{H}}$ : contrainte hydrostatique locale.

Cette relation permet de prévoir une augmentation de la solubilité en présence d'une contrainte hydrostatique de traction et une diminution pour une contrainte de compression [23, 29].

\subsection{Diffusion et perméation de l'hydrogène}

Du fait de sa faible solubilité la diffusion interstitielle de l'hydrogène dans les alliages base Fe et Ni s'effectue selon les mécanismes classiques d'hétérodiffusion à dilution infinie. Il est ainsi possible de modéliser la pénétration (ou la désorption) de l'hydrogène à partir de la résolution des équations de Fick 
Tableau 2. Lois de variation, en fonction de la température, de la solubilité, la diffusion et la perméation de l'hydrogène dans des alliages base $\mathrm{Fe}$ et $\mathrm{Ni}\left(\mathrm{R}\right.$ en $\mathrm{J} \cdot \mathrm{K}^{-1} \cdot \mathrm{mol}^{-1}$, $\mathrm{p}$ en atm).

\begin{tabular}{|c|c|c|c|}
\hline & $\begin{array}{c}\text { Solubilité } \\
\text { (ppm masse })\end{array}$ & $\begin{array}{c}\text { Diffusion } \\
\left(\mathrm{cm}^{2} \cdot \mathrm{s}^{-1}\right)\end{array}$ & $\begin{array}{c}\text { Perméation } \\
\left(\mathrm{cm}^{3} \cdot \mathrm{s}^{-1} \cdot \mathrm{cm} \cdot \mathrm{cm}^{2} \cdot \mathrm{atm}^{1 / 2}\right)\end{array}$ \\
\hline Fera & $S=33 p^{1 / 2} \exp \left(\frac{-28600}{R T}\right)$ & $D=5,12.10^{-4} \exp \left(\frac{-4150}{R T}\right)$ & $P=5,77.10^{-3} p^{1 / 2} \exp \left(\frac{-34320}{R T}\right)$ \\
\hline Aciers & $S=33,1 p^{1 / 2} \exp \left(\frac{-11080}{R T}\right)$ & $D=5,8.10^{-3} \exp \left(\frac{-53800}{R T}\right)$ & $P=1,89.10^{-2} p^{1 / 2} \exp \left(\frac{-65040}{R T}\right)$ \\
\hline inoxydables g & $S=57,8 p^{1 / 2} \exp \left(\frac{-15466}{R T}\right)$ & $D=4,0.10^{-3} \exp \left(\frac{-39250}{R T}\right)$ & $P=2,3.10^{-2} p^{1 / 2} \exp \left(\frac{-54716}{R T}\right)$ \\
\hline Nickel & $S=10,1 p^{1 / 2} \exp \left(\frac{-6855}{R T}\right)$ & $D=4,7.10^{-3} \exp \left(\frac{-46607}{R T}\right)$ & $P=4,3.10^{-3} p^{1 / 2} \exp \left(\frac{-53462}{R T}\right)$ \\
\hline $\begin{array}{c}\text { Alliage base } \\
\text { Ni }\end{array}$ & $S=10$ & \\
\hline
\end{tabular}

pour différentes conditions aux limites [30, 31]. Les deux grandeurs utilisées de façon pour caractériser la diffusion sont :

- le coefficient de diffusion $\mathrm{D}\left(\mathrm{m}^{2} \cdot \mathrm{s}^{-1}\right)$ dont la variation avec la température s'exprime par une loi d'Arrhénius :

$$
\mathrm{D}=\mathrm{D}_{\mathrm{o}} \exp \left(\frac{-\Delta \mathrm{H}_{m}}{k T}\right)
$$

$\Delta \mathrm{H}_{m}$ : enthalpie de migration

- la perméabilité $\mathrm{P}\left(\mathrm{cm}^{3} \cdot \mathrm{cm}^{-2} \cdot \mathrm{s}^{-1}\right)$ qui caractérise le flux stationnaire mesuré dans des conditions données au travers d'une paroi d'épaiseur $(1 / \mathrm{K})$ connue. Son expression en fonction de la température et de la pression peut être déduite de celle de $\mathrm{S}$ et $\mathrm{D}$ :

$$
\mathrm{P}=\mathrm{KS}_{\mathrm{o}} D_{\mathrm{o}} \Delta p^{1 / 2} \exp -\frac{\left(\Delta H_{s}+\Delta H_{m}\right)}{k T}
$$

Le coefficient de perméabilité (généralement exprimé en $\mathrm{cm}^{3} \cdot \mathrm{cm} \cdot \mathrm{cm}^{-2} \cdot \mathrm{s}^{-1} \cdot \mathrm{atm}^{-1 / 2}$ ) est une grandeur caractéristique du matériau qui permet de s'abstraire du rôle de la pression et de la géométrie de la paroi.

\subsubsection{Données expérimentales sur la diffusion et la perméabilité de l'hydrogène}

Le tableau 2 rassemble quelques lois de variation, en fonction de la température, des coefficients de diffusion et de perméabilité de l'hydrogène dans le fer $\alpha$, les aciers inoxydables austénitiques, le nickel et un alliage base $\mathrm{Ni}$. Ces données montrent qu'il existe au voisinage de la température ambiante des différences considérables de mobilité de l'hydrogène en fonction de la structure cristalline du matériau (environ 7 ordres de grandeurs pour la diffusion entre les structures $\alpha$ et $\gamma$ des aciers et 5 ordres de grandeurs pour le coefficient de perméabilité). Ceci est à prendre en compte pour prédire et interpréter les effets de température et de microstructure sur le comportement en présence d'hydrogène des matériaux [34].

\subsubsection{Autres formes de transport de l'hydrogène}

Les phénomènes de court-circuit de diffusion associés à la présence de défauts linéaires (dislocations) ou planaires (joints de grain) ne sont pas observés pour l'hydrogène lorsque son coefficient de diffusion réticulaire est très élevé $(\mathrm{Fe} \alpha)$. En revanche des phénomènes de diffusion préférentielle le long des joints de grains ou des structures de dislocations ont été mis en évidence à température ambiante dans certaines structures austénitiques stables [35-39]. 
De la même façon le phénomène de transport de l'hydrogène par les dislocations mobiles a été mis en évidence expérimentalement dans les structures cfc peu perméables à l'hydrogène [40-43]. Ce phénomène sera discuté plus en détail plus loin (cf. §4).

\subsection{Piégeage de l'hydrogène par les défauts microstructuraux}

Le phénomène de piégeage et son influence sur la diffusion et la fragilisation par l'hydrogène a fait l'objet de nombreuses études dans le cas des alliages base fer [26, 44-49].

\subsubsection{Origine du piégeage}

Le piégeage a pour origine l'existence d'une force de transport externe qui modifie la probabilité de saut dans une direction donnée ou d'une perturbation locale du réseau qui modifie la fréquence de saut [44]. Au niveau d'un site de piégeage l'hydrogène pourra diminuer son potentiel chimique. Du point de vue cinétique, l'équilibre entre l'hydrogène occupant un site de piégeage et l'hydrogène dans un site interstitiel est défini par des constantes de vitesse de piégeage et de dépiégeage. La barrière énergétique étant plus haute dans le sens piège-réseau, la probabilité de saut dans ce sens sera réduite et la diffusion en présence de piège sera ralentie.

Différents critères sont utilisés pour une classification du type de piégeage : la localisation du piégeage, son origine physique, l'existence ou non d'une interaction à longue distance entre le piège et l'atome interstitiel, sa nature physique, la valeur de l'énergie d'interaction hydrogène-piège.

Pour une température donnée, il est important d'établir une distinction entre les pièges "irréversibles" c'est à dire au niveau desquels la probabilité de dépiégeage est quasi nulle et les pièges "réversibles", d'énergie plus faible, pour lesquels il existe un équilibre entre les atomes d'hydrogène qui occupent le piège et ceux qui occupent les sites du réseau.

\subsubsection{Grandeurs caractéristiques du piégeage}

Dans un métal pour lequel les grandeurs $\Delta \mathrm{H}_{\mathrm{m}}$ et $\Delta \mathrm{H}_{\mathrm{s}}$ sont connues, les pièges sont définis par leur énergie $\Delta \mathrm{H}_{\mathrm{b}}$, leur densité (nombre de sites par unité de volume), les vitesses de piégeage et de dépiégeage $\left(\mathrm{k}_{1}\right.$ et $\left.\mathrm{k}_{-1}\right)$ et leur taux d'occupation $\mathrm{c}$.

Quelques valeurs d'énergie de piégeage estimées par différents auteurs pour différents défauts microstructuraux sont rassemblées dans le tableau 3 [50]. La forte dispersion des valeurs pour un même type de défaut microstructural illustre la grande incertitude qui subsiste aussi bien dans l'identification des sites de piégeage que dans la mesure de la valeur d'énergie de liaison.

En négligeant le terme entropique et à l'aide de l'expression de la solubilité de l'hydrogène (\$2.3) l'équilibre H-piège peut s'exprimer en fonction de la pression et de la température, pour une solution solide très diluée, sous la forme [26] :

$$
\frac{\mathrm{c}}{1-\mathrm{c}}=S_{o} p^{1 / 2} \exp \left[\frac{\left(\Delta H_{b}-\Delta H_{s}\right)}{k T}\right]
$$

Le taux d'occupation des pièges dans le fer a été calculé pour différentes énergies de liaison caractéristiques de divers défauts microstructuraux et différentes conditions de température et de pression [26].

\subsubsection{Diffusion en présence de pièges}

La diffusion interstitielle en présence de pièges s'exprime sous la forme générale :

$$
\frac{\delta \mathrm{c}_{\mathrm{r}}}{\delta \mathrm{t}}+\frac{\delta \mathrm{c}_{p}}{\delta \mathrm{t}}=D \nabla^{2} c_{r}
$$

$\mathrm{c}_{r}$ et $\mathrm{c}_{p}$ : concentration en $\mathrm{H}$ dans les sites interstitiels et dans les pièges respectivement, $\mathrm{t}:$ temps. 
Tableau 3. Énergies de piégeage de l'hydrogène avec différents défauts microstructuraux dans les alliages base $\mathrm{Fe}$ ou Ni.

\begin{tabular}{|l|c|c|}
\hline & \multicolumn{2}{|c|}{$\begin{array}{c}\text { Énergie de liaison } \Delta H_{\mathrm{b}} \\
\langle\mathrm{kJ} / \mathrm{mol}\rangle\end{array}$} \\
\hline Nature du site & $\begin{array}{c}\text { Fer ou aciers } \\
\text { ferritiques }\end{array}$ & $\begin{array}{c}\text { Aciers austénitiques } \\
\text { ou nickel }\end{array}$ \\
\hline Dislocation & 20 à 58 & 10 à 20 \\
\hline Joint de grains & 10 à 58 & 10 à 20 \\
\hline & $\mathrm{MnS}: 29$ à 57 & \\
\hline Interfaces (précipiteś, & $\mathrm{AIN}: 48$ à 58 & \\
\hline inclusions) & $\mathrm{Fe}_{3} \mathrm{C}: 57$ à 84 & 20 à 42 \\
\hline & $\mathrm{TiC}: 95$ & 41 à 53 \\
\hline Lacune & 46 à 54 & \\
\hline Surface interne, cavité & 28 à 96 & \\
\hline
\end{tabular}

De nombreux modèles ont été développés pour tenter de déterminer à partir de mesures de perméabilité les grandeurs caractéristiques du piégeage [46-49] ; un grand nombre d'hypothèses simplificatrices leurs sont généralement associées. Dans le cas du modèle simple d'Oriani [46] qui permet d'établir une relation entre le coefficient de diffusion $\mathrm{D}_{\mathrm{app}}$ déterminé expérimentalement à partir du régime transitoire de perméation et le coefficient de diffusion réticulaire $\mathrm{D}_{\mathrm{r}}$ correspondant à une diffusion sans piégeage, les principales hypothèses sont les suivantes :

- un seul type de piège, une seule énergie de piégeage $\Delta \mathrm{G}_{p}$ indépendante du taux d'occupation $\theta_{p}$, un seul atome par site,

- transport d'hydrogène par diffusion interstitielle, densité de sites interstitiels $\mathrm{N}_{r}$ très grande par rapport à celle des pièges $\mathrm{N}_{p}$, faible concentration en hydrogène interstitiel $\mathrm{c}_{r}$

- absence d'interaction entre sites de piégeage, mais équilibre local $\mathrm{H}_{\text {piégé }} / \mathrm{H}_{\text {interstitiel}}$,

- flux stationnaire d'hydrogène indépendant du piégeage.

En résumé les phénomènes de piégeage ont pour effet d'augmenter la solubilité apparente de l'hydrogène, de diminuer sa diffusivité apparente, d'introduire une éventuelle dépendance de la cinétique de pénétration de l'hydrogène vis à vis de son activité sur la surface et de favoriser des phénomènes de surconcentration locale en hydrogène. Ces effets peuvent être soit bénéfiques soit défavorables en ce qui concerne la sensibilité à la fragilisation par l'hydrogène du matériau. Le piégeage peut jouer un rôle favorable en limitant l'entrée de l'hydrogène dans le matériau et en réduisant l'activité de l'hydrogène diffusible ; il peut au contraire favoriser l'amorçage de fissure dans les zones fortement enrichies en hydrogène. Ces effets antagonistes dépendent en particulier de l'énergie et de la distribution des pièges dans la microstructure.

Enfin il est important d'introduire la notion de «piège mobile ». Cela concerne particulièrement les dislocations impliquées dans les interactions H-plasticité. Le mouvement des dislocations dans un réseau métallique contenant de l'hydrogène en solution solide peut, par un effet de traînage élastique [51], et en raison du maintien d'un équilibre entre $H$ piégé reversiblement et $H$ dans le réseau, entraîner un transport de l'hydrogène. Cet effet de «dragging » et ses conséquences sur l'endommagement des matériaux sera discuté plus loin (cf. §4.).

\section{PRINCIPAUX EFFETS D’ENDOMMAGEMENT ASSOCIÉS À L'ABSORPTION D'HYDROGÈNE}

L'absorption d'hydrogène dans les matériaux métalliques conduit à différentes formes d'endommagement associées à trois grandes familles de mécanismes fondés sur : des transformations de phases, des processus de décohésion ou des interactions hydrogène-déformation. La 
complexité des phénomènes observés dans la réalité est souvent la conséquence de la combinaison de plusieurs mécanismes.

\subsection{Fragilisation induite par la précipitation d'hydrures}

La précipitation d'un hydrure associée à une sursaturation (locale) en hydrogène dans des conditions de température et de pression données et/ou assistée par un champ de contrainte peut induire une forte fragilité de certains matériaux [52]. Ce mécanisme ne concerne les alliages base fer et nickel que pour des conditions très particulières $[34,53]$.

\subsection{Fragilisation par un mécanisme de décohésion}

L'hydrogène peut être responsable de l'amorçage et la propagation de fissures en favorisant différents types de décohésion du réseau cristallin ou des interfaces.

\subsubsection{Décohésion associée à des fortes activités d'hydrogène}

En présence d'une très forte pression équivalente d'hydrogène imposée en surface $(\$ 2.3)$ ou sous l'effet d'une sursaturation associée au brusque changement de température d'un matériau contenant une quantité élevée d'hydrogène, différentes formes de fissuration des aciers peuvent être observées en l'absence de contrainte imposée : cloquage, fissuration interne, disbonding, ... [54]. Cette fissuration résulte d'une recombinaison de l'hydrogène atomique en hydrogène moléculaire sur des défauts (microcavités,...) qui jouent le rôle de pièges $[55,56]$. Ces phénomènes sont observés pour des conditions très sévères d'exposition des aciers à l'hydrogène, notamment dans l'industrie pétrolière (milieu $\mathrm{H}_{2} \mathrm{~S}$ ) [57]. L'amplitude de la fissuration est également fortement dépendante de la présence de contraintes internes et de la microstructure du matériau (propreté inclusionnaire, nature, densité et répartition des sites de piégeage, ...).

\subsubsection{Diminution de l'énergie de surface}

La diminution de l'énergie d'une surface métallique sous l'effet de l'adsorption d'atomes d'hydrogène est fondée sur la relation de Gibbs [58]. Ce phénomène a pour effet de diminuer la contrainte nécessaire à la propagation d'une fissure exposée à un environnement source d'hydrogène [59]. Il nécessite un taux de recouvrement en hydrogène élevé, donc une température modérée.

\subsubsection{Décohésion associée aux interactions électroniques hydrogène/métal}

L'absorption d'hydrogène conduit à un affaiblissement des liaisons métal-métal par modification de l'environnement électronique des atomes [4, 60-62]. Ce phénomène, qui est à l'origine de l'effet fragilisant de l'hydrogène, est d'autant plus marqué que la concentration locale en hydrogène est grande. Il est généralement associé à l'augmentation de la concentration locale en hydrogène dans des champs de contrainte hydrostatique. Il permet d'expliquer la rupture différée des aciers à hautes caractéristiques mécaniques et certains phénomènes de propagation de fissure assistée par l'hydrogène.

\subsection{Fragilisation associée aux interactions hydrogène-dislocations}

Nous nous limitons ici à quelques généralités sur les interactions hydrogène-plasticité et leurs conséquences qui sont présentées par ailleurs en détail dans le cadre de cette école [63, 64]. Ces interactions impliquent à la fois un effet de l'hydrogène sur le mouvement des dislocations et un effet des dislocations sur le transport de l'hydrogène qui peuvent tous les deux contribuer à l'endommagement du matériau. 


\subsubsection{Ségrégation d'hydrogène assistée par les dislocations mobiles}

L'existence d'un transport de l'hydrogène par les dislocations [65] est fondée sur le phénomène de piégeage de l'hydrogène dans le coeur et le champ de contrainte des dislocations. On admet l'existence, dans certaines conditions, d'une ségrégation d'hydrogène associée au mouvement collectif des dislocations. La surconcentration locale qui en résulte, combinée à la présence de fortes contraintes associées aux empilements de dislocations peut promouvoir l'amorçage et/ou la propagation d'une fissure. L'accumulation locale d'hydrogène est favorisée par un mouvement coplanaire des dislocations et dépend de la nature et la distribution des défauts dans la microstructure. Ces derniers peuvent en effet limiter le mouvement des dislocations ou encore réduire par effet de piégeage la quantité d'hydrogène transportée. Au contraire certains pièges réversibles peuvent se comporter comme des sources pour les dislocations mobiles [50]. La microstructure du matériau joue donc un rôle important.

\subsubsection{Plasticité locale induite par l'hydrogène}

Ce phénomène, décrit en détail par ailleurs [51, 66-70], résulte de l'existence d'une interaction de l'hydrogène avec le champ de contrainte des dislocations. Il impose l'existence d'une activité élevée de l'hydrogène dans le réseau. L'augmentation locale de plasticité peut, au plan macroscopique, contribuer à une perte des propriétés mécaniques du matériau.

\subsection{Fragilisation associée à une réduction des impuretés métalloïdiques : « attaque hydrogène »}

Ce mécanisme d'endommagement concerne particulièrement les aciers ferritiques exposés à température élevée (au delà de $300^{\circ} \mathrm{C}$ ) à de fortes pressions d'hydrogène [71, 72]. Il résulte de la forte réactivité chimique entre l'hydrogène en solution dans le métal et divers métalloïdes $(\mathrm{C}, \mathrm{O}, \mathrm{N}, \ldots)$ présents dans les aciers. Elle conduit à la formation de composés $\left(\mathrm{CH}_{4}, \mathrm{H}_{2} \mathrm{O}, \mathrm{NH}_{3}\right)$ qui précipitent sous forme de bulles sur certains défauts microstructuraux et fragilisent l'acier. La composition chimique de l'acier et sa microstructure sont les paramètres déterminants pour ce type de fragilisation.

\subsection{Fragilisation par l'hélium 3}

La fragilisation par l'hélium 3 constitue une forme particulière de fragilisation par un isotope de l'hydrogène (le tritium) dont la décroissance radioactive donne naissance à de l'hélium 3 [73]. Ainsi le vieillissement d'un acier contenant du tritium conduit à la formation de bulles d'hélium sur les défauts structuraux du métal dès que les conditions seront favorables d'un point de vue cinétique. Cette précipitation, qui peut avoir lieu lorsque la température de vieillissement du matériau dépasse $650^{\circ} \mathrm{C}$, conduit généralement à une fragilité intergranulaire.

Pour tous les mécanismes cités précédemment la microstructure et les interactions hydrogènedéformations [74] jouent un rôle déterminant dans le processus de fragilisation (précipitation d'hydrure ou enrichissement local en hydrogène dans les champs de contrainte, interactions hydrogènedislocations, ...). La déformation peut avoir également pour effet de favoriser l'entrée de l'hydrogène dans le matériau par rupture du film superficiel d'oxyde ou de passivation, ou de favoriser la formation dans l'acier de phases présentant une plus forte sensibilité à la fragilisation par l'hydrogène (cas des phases martensitiques formées dans les aciers inoxydables austénitiques instables [34]).

L'effet fragilisant de l'hydrogène rencontré dans la pratique est fréquemment une combinaison de mécanismes du type de ceux présentés ci-dessus qui interviennent à la fois sur l'entrée de l'hydrogène dans le matériau et sur son endommagement. 


\section{QUELQUES QUESTIONS ET REMARQUES CONCERNANT LES INTERACTIONS HYDROGÈNE-PLASTICITÉ}

Les interactions plasticité-environnement impliquent l'existence de contraintes d'élasticité et d'un relachement des contraintes par déformation plastique qui génère à la fois des défauts ponctuels et des dislocations. Se posent alors différentes questions concernant le rôle de l'état de contrainte-déformation à l'interface métal/environnement et dans le volume du matériau sur le comportement de l'hydrogène.

\subsection{Influence d'un champ de contrainte élastique}

\subsubsection{Influence de la contrainte sur le coefficient de diffusion}

Les quelques études de perméation consacrées à l'influence de contraintes hydrostatiques montrent l'absence d'un effet significatif des contraintes sur le coefficient de diffusion [75]. De la même façon il existe très peu de données sur la valeur du volume d'activation de l'hydrogène dans les matériaux de stucture. Sa valeur est vraisemblablement proche de zéro.

\subsubsection{Influence de la contrainte sur la solubilité}

La dépendance de la solubilité de $\mathrm{H}$ vis à vis de la contrainte présentée \$2.3, résulte de la valeur élevée du volume partiel molaire de l'hydrogène dans la plupart des métaux et alliages. A cette variation de solubilité est directement associée une variation du flux de perméation de l'hydrogène dans le matériau sous contrainte [75].

\subsubsection{Influence de la contrainte sur les mécanismes de transfert à l'interface métal/environnement}

Le rôle de la contrainte sur les mécanismes de dissociation de la molécule d'hydrogène comme sur les mécanismes de décharge et recombinaison de l'hydrogène cathodique est encore mal connu bien qu'il ait été montré qu'un champ de contrainte élastique affecte la cinétique de la réaction de décharge cathodique [76]. Ces phénomènes de surface risquent de modifier les conditions aux limites de la diffusion en volume.

\subsection{Influence d'une déformation plastique}

Peu d'études ont été consacrées aux interactions H-défauts ponctuels. Nous nous limiterons ici aux interactions $\mathrm{H}$-dislocations en distinguant entre les défauts mobiles et immobiles.

\subsubsection{Interactions $H$-dislocations statiques}

A l'interface métal/environnement, la déformation plastique augmente la cinétique de la réaction de décharge cathodique [76, 77]. Ce phénomène paraît étroitement lié à la structure de dislocations fonction du taux de déformation du matériau [77].

En volume, les dislocations statiques peuvent jouer un double rôle de site de piégeage (cf. §2.5) et de chemin préférentiel de diffusion (pipe diffusion). Si le piégeage de $\mathrm{H}$ dans le champ de contrainte élastique d'une dislocation coin est bien documenté, il existe en revanche peu de données précises sur les interactions $\mathrm{H}$-cœur de dislocations. L'augmentation de densité de dislocations associée à une déformation plastique conduit, par effet de piégeage, à une diminution du coefficient de diffusion apparent et une augmentation de la concentration en hydrogène piégé. Cependant il existe encore très peu de données précises concernant la valeur à prendre en compte pour le nombre d'atome d'hydrogène par unité de longueur de dislocation, dans un calcul de la variation de la quantité d'hydrogène piégé associée à la variation de la densité de dislocation dans le matériau déformé. L'intérêt de ce type de 
calcul résulte des changements importants de concentration en hydrogène réticulaire (donc diffusible) qui peuvent être associés à un accroissement de la densité de sites de piégeage. En effet, en système « fermé » $\left(\mathrm{C}_{\mathrm{H}}^{\text {total }}\right.$ constant $)$, l'augmentation de la densité de sites de piégeage conduit à une diminution de la concentration en hydrogène réticulaire susceptible d'interagir avec des dislocations mobiles ou de ségréger rapidement dans des champs de contrainte/déformation. Ce phénomène peut à la fois affecter la plasticité du matériau (effet de $\mathrm{H}$ sur le mouvement des dislocations) et la ségrégation de l'hydrogène (effet sur le transport d'hydrogène). Il peut intervenir localement dans un zone plastique en tête de fissure et affecter la cinétique de propagation de la fissure.

L'amplitude du phénomène de «pipe diffusion » est vraisemblablement associée à la présence d'un réseau plus ou moins dense et continu de dislocations. Ce phénomène mériterait d'être caractérisé de façon plus précise en relation avec la structure de dislocation du matériau [36].

\subsubsection{Interactions H-dislocations mobiles}

Les dislocations mobiles peuvent participer, à température ambiante, à un mécanisme de traînage élastique qui se traduit par un dragage de soluté. Des approches simplifiées ont été utilisées pour caractériser, pour des conditions $\left(\mathrm{T}, \mathrm{a}_{\mathrm{H}}\right)$ données, la capacité de transport d'une dislocation à l'aide du taux d'occupation du piège (cf. §2.5) et de la vitesse critique moyenne au delà de laquelle le transport n'est plus effectif [78-80]. Ce phénomène de transport accéléré est bien mis en évidence dans différentes études expérimentales [36, 40-43]. Cependant les fondements physiques d'un transport « longue distance » dans les matériaux polycristallins restent à préciser en relation avec un effet probable du mouvement collectif des dislocations.

De la même façon, l'existence d'une surconcentration locale transitoire associée au mouvement des dislocations, proposée pour interpréter la rupture intergranulaire d'alliages base nickel [80], reste à mettre en évidence expérimentalement et à modèliser pour valider le rôle du transport de l'hydrogène dans le mécanisme de fragilisation.

\section{Références}

[1] Chêne J. and Brass A.M., Corrosion sous contrainte, Eds. D. Desjardins, R. Oltra, Les Editions de Physique, 1992, pp. 159-210.

[2] Alefeld G. and Völkl J., Hydrogen in metals (Springer verlag, Berlin, 1978) pp. 321.

[3] Baskes M.I., Melius C.F. and Wilson W.D., Hydrogen Effects in Metals, Eds. I. Bernstein, A. Thompson, TMS AIME, 1980 pp. 67-75.

[4] Itsumi Y. and Ellis D.E., J. Mater. Res. 11 (1996) 2206.

[5] Speiser R., Stress corrosion cracking and hydrogen embrittlement of iron base alloys (NACE Pub., Unieux, France, 1973) p. 226-243.

[6] Feaugas X., Ecole Plastox (Mai 2007).

[7] Guiraldenq P., Techniques de l'Ingénieur M 55, vol. M1I.

[8] Brass A.M., Ann. Chim. Fr. 14 (1989) 273.

[9] Debnath N.C. and Anderson A.B., Surf. Sci. 128 (1983) 61.

[10] Brass A.M., Boutry-Forvielle A. and Aucouturier M., J. Electrochem. Soc. 139 (1992) 374.

[11] Wultz H. and Fromm E., J. Less Common Met. 118 (1986) 293.

[12] Clermont R., Aucouturier M., Brass A.M., Faudou J.Y and Guenot, Hydrogène dans les matériaux (Pergamon Press, Paris, 1977, 1A2) pp. 1-8.

[13] Tison P., Thèse Univ. Paris 6, Rapport CEA R 5240 (1984).

[14] Defranoux J.M., Techniques de l'ingénieur M 150, vol. M1II.

[15] Brass A.M. and Collet-Lacoste J., "Hydrogen Transport and Cracking in Metals", A. Turnbull Ed, The Institute of Materials (1995) pp. 142-154.

[16] Bockris J.O’M. and Subramanyan P.K., Electrochim. Acta 16 (1971) 2169. 
[17] Bockris J.O'M. and Mauser H., Corr. J. Chem. 37 (1971) 475.

[18] Frumkin A.N., J. Electrochem. Soc. 107 (1960) 461.

[19] Parsons R., Trans. Faraday Soc. 47 (1951) 1331.

[20] Bockris J.O'M., Mc Breen J. and Nanis, J. Electrochem. Soc. 112 (1965) 1025.

[21] Berkowitz B.J. and Horowitz H.H., J. Electrochem. Soc. 129 (1982) 468.

[22] Newman J.F. and Shreir Corr. Sci. 9 (1969) 631.

[23] Bockris J.O'M., Stress corrosion cracking and hydrogen embrittlement of iron base alloys (NACE Pub., Unieux, Fr, 1973) pp. 286-305.

[24] Protopopoff E. and Marcus P., "Electrochemistry and Materials Science", PV 94-21 Electrochemical Soc. Proc. Séries B.E. Conway, G. Jierkiewicz Eds., (Pennington N.J., 1995), p. 374.

[25] Baranowski B., Ber. Bunsenges. Physik. 76 (1972) 714.

[26] Hirth J.P., Met. Trans. A 11 1980, 861.

[27] Interrante C.G., "Current solutions to hydrogen problem in steels" ASM., C. G. Interrante, G.M. Pressouyre, Eds. (1982) p. 3-17.

[28] Brass A.M., Thèse Université Paris-Sud, 1983.

[29] Oriani R.A., Am. Rev. Mater. Sci. 8 (1978) 327.

[30] Crank J., The mathematics of diffusion (Clarendon Press, Oxford, 1975).

[31] Philibert J., Diffusion et transport de matière dans les solides (Les Editions de Physique, Paris, 1985).

[32] Riecke E. and Bohnenkamp K., Z. Metallkde 76 (1984) 76.

[33] Riecke E., Werkstoffe und Korrosion 32, 66 (1981) 66.

[34] Chêne J., "Current solutions to hydrogen problem in steels" ASM, C.G. Interrante, G.M. Pressouyre, Eds. (1982) pp. 263-271.

[35] Chêne J., Brass A.M., Trabuc P. and Gastaldi O., J. Nucl. Mater. 360 (2007) 177.

[36] Brass A.M. and Chêne J., Corrosion Science 48 (2006) 3222.

[37] Brass A.M., Chanfreau A. and Chêne J., "Hydrogen effects on materials behavior" TMS, N.R. Moody and A.W. Thompson, Eds. (1990) pp. 19-31.

[38] Brass A.M., and Chanfreau A., Acta Mater. 44 (1996) 3823.

[39] Calder R.D., Elleman T.S., and Verghese K., J. Nucl. Mat. 46 (1973) 46.

[40] Donovan J.A., Met. Trans. A 7 (1976) 1677.

[41] Louthan M.R., Caskey G.R., Donovan J.A. and Rawl D.E., Mat. Sci. Eng. 10 (1972) 357.

[42] Chêne J., Aucouturier M., Arnoult-Laurent R., Tison P. and Fidelle J.P., "Hydrogen Effects in Metals", TMS AIME, I.M. Bernstein, A.W. Thompson, Eds. (1980) pp. 583-595.

[43] Chêne J. and Brass AM., Scripta Materialia 40 (1999) 537.

[44] Pressouyre G.M., Acta Met. 28 (1979) 895.

[45] Mc Nabb A. and Foster P., Trans. Met. Soc. 227 (1963) 618.

[46] Oriani R.A., Acta Met. 18 (1970) 147.

[47] Caskey G. and Pillinger, Met. Trans. A 6 (1975) 467.

[48] Iino M., Acta Met. 28 (1979) 895.

[49] Leblond, J.B. and Dubois D., Acta Met. 331 (1983) 1459.

[50] Brass A.M., Chêne J. and Coudreuse L., Techniques de l'Ingénieur, traité Matériaux métalliques M176 (2000) 1.

[51] Girardin G., Thèse ENSM Saint-Etienne-Univ. Jean Monnet Saint-Etienne (2005).

[52] Birnbaum H.K., "Hydrogen embrittlement and stress corrosion cracking" ASM, R. Gibala, R. Hehemann, Eds. (1984) 153.

[53] Narita N., Altstetter C. and Birnbaum H.K., Met. Trans. A 13 (1982) 1355.

[54] Brass A.M., Chêne J. and Coudreuse L., Techniques de l'Ingénieur, traité Matériaux métalliques, 175 (2000) 1.

[55] Zapffe C. and Sims C.T., Trans. AIME 145 (1941) 225. 
[56] Iino M., "Hydrogen degradation of ferrous alloy" R.A. Oriani, J.P. Hirth, M. Smialowski, Eds. (Noyes publications, 1985) pp. 737-761.

[57] Ropital F., Ecole Plastox (Mai 2007).

[58] Gibbs J.W., The collected works of J.W. Gibbs (Yale Univ. Press, New Haven, 1957).

[59] Petch N.J., Phil. Mag. 1, 8ème série (1966) 331.

[60] Troiano A.R., Trans. ASM 52 (1960) 54.

[61] Messmer R.P. and Briant C.L., Acta Met. 30 (1982) 457.

[62] Daw M.S. and Baskes M.I., Phys. Rev. B 29 (1984) 6443.

[63] Delafosse D., Ecole Plastox (Mai 2007).

[64] Tanguy D., Ecole Plastox (Mai 2007).

[65] Bastien P. and Azou P., Revue de Métallurgie 12 (1952) 837.

[66] Beachem C.D., Met. Trans. 3 (1972) 437.

[67] Birnbaum H.K., "Hydrogen effects on material behavior", Eds N.R. Moody, A.W. Thompson, TMS pub., (1990) pp. 639-660.

[68] Sofronis P. and Birnbaum H.K., J. Mech. Phys. Solids 43, n 1 (1995) 49.

[69] Lynch S.P., Acta Met. 36 (1988) 2639.

[70] Chateau J.P., Thèse ENSM Saint Etienne-INPG Grenoble n 202 TD (1999).

[71] Nelson G.A., Proc. API 45 (1965) 190.

[72] Shewman P.G., Met. Trans. A 7 (1976) 279.

[73] Ullmaier H., Nucl. Fusion 24 (1984) 1039.

[74] Magnin T., "Advances in Corrosion-Deformation Interactions", Materials Science Forum, Vol. 202 (Trans. Tech. Pub., 1996) pp. 125-130.

[75] Beck W., Bockris J.O’M., Mc Breen J., and Nanis L., Proc. Roy. Soc. London 290 (1966) 125.

[76] Despic A.R., Raicheff R.G. and Bockris J.O’M., J. Chem. Phys. 49 (1968) 926.

[77] El Alami A., Thèse Univ. La Rochelle (2007).

[78] Tien J.K., Nair S.V. and Jensen R.R., "Hydrogen Effects in Metals", Eds I.M. Bernstein, A.W. Thompson, TMS AIME, 1980, pp. 37-56.

[79] Hashimoto M. and Latanision R.M., Acta Metal. 36 (1988) 1837.

[80] Chêne J. and Brass A.M., Met. Mater. Trans. A 35 (2004) 457. 
